\title{
Arterial hypertension and remodelling of the right ventricle
}

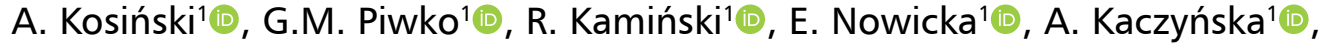

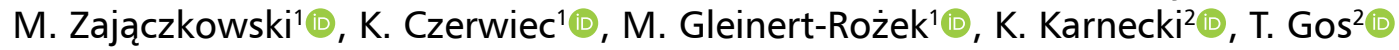 \\ ${ }^{1}$ Department of Clinical Anatomy, Gdansk Medical University, Gdansk, Poland \\ 2Department of Forensic Medicine, Gdansk Medical University, Gdansk, Poland
}

[Received: 19 December 2020; Accepted: 11 March 2021; Early publication date: 13 April 2021]

\begin{abstract}
Background: In the case of long-term and physiological loads (e.g. during pregnancy or regular athletics training), reversible morphological changes occur in the heart - cardiomyocytes undergo hypertrophy; however, this is not accompanied by impairment of left ventricular function or myocyte metabolism. However, in the course of various pathological processes, as time goes by, gradually permanent morphological changes occur. These changes are referred to as remodelling of the heart muscle, which, regardless of the primary cause, can lead to the development of chronic heart failure.

Materials and methods: The study was performed on post-mortem material of 35 human hearts obtained from forensic sections and anatomopathological sections of people who died of non-cardiac causes (mainly traffic accidents, suicide attempts, strokes, acute infections); material was fixed in a 4\% formalin solution. The hearts were subjected to macro- and microscopic assessment. During microscopic assessment the features of remodelling were evaluated.

Results and Conclusions: In vivo and echocardiographic tests, as well as macroscopic evaluation of post-mortem material, suggest the presence of some kind of right ventricular muscle remodelling; however, classic microscopic observations, presented in this study, do not provide such unambiguous evidence. Thus, the question arises: why and how the right ventricular function is disturbed, sometimes at early stages of arterial hypertension. (Folia Morphol 2022; 81, 2: 336-342)
\end{abstract}

Key words: heart, human, changes, hypertensive

\section{INTRODUCTION}

Various pathological factors, both external and intracardiac, can negatively affect the effectiveness of the heart. In return, the organ reacts to these factors by changes in its structure and function, which are aimed at ensuring efficient work in altered, sometimes adverse conditions. In the time of well-being, the heart has a wide range of compensation mechanisms. In the case of short-term physiological loads, the heart muscle mainly uses the mechanisms of: a) homeometric regulation - an increase in contractility without changing the sarcomere length (Anrep effect) and an increase in contractility with an increase in heart rate (Bowditch effect) [1]; b) heterometric regulation - an increase in contractility associated with an increase in the length of sarcomeres (Frank-Starling law) [14]. In the case of longterm and physiological loads (e.g. during pregnancy

Address for correspondence: Dr. G.M. Piwko, Department of Clinical Anatomy, ul. Dębinki 1, 80-210 Gdańsk, Poland, tel: +48 58349 14 20, e-mail: grzegorz.piwko@gumed.edu.pl

This article is available in open access under Creative Common Attribution-Non-Commercial-No Derivatives 4.0 International (CC BY-NC-ND 4.0) license, allowing to download articles and share them with others as long as they credit the authors and the publisher, but without permission to change them in any way or use them commercially. 
or regular athletics training), reversible morphological changes also occur in the heart - cardiomyocytes undergo hypertrophy: however, this is not accompanied by impairment of left ventricular (LV) function or myocyte metabolism $[7,12,20]$. In the course of various pathological processes, as time passes, the basic compensatory mechanisms become exhausted, and gradually lead to permanent morphological changes $[4,10,27]$. Within the myocardium, the proportions and correlation between myocytes, fibroblasts and endothelial cells are disturbed. In the literature, the above changes are referred to as remodelling of the LV heart muscle, which, regardless of the primary cause (hypertension, hypertrophic cardiomyopathy, valvular heart disease, ischaemic disease, etc.), can lead to the development of chronic heart failure, or right ventricular (RV) hypertrophy with all its adverse consequences when appropriate factors affect right ventricle by causing pulmonary hypertension (e.g. chronic obstructive pulmonary disease, idiopathic pulmonary hypertension, chronic thromboembolic pulmonary hypertension, mitral valve insufficiency, etc.).

Due to the increasing prevalence of hypertension in overall population, this group of patients (almost $60 \%$ of the population $>59$ years of age) is particularly at risk. Additionally, if we take into account the fact that only about $26 \%$ of treated patients manage to achieve the intended therapeutic effect, it is not surprising that hypertension and its complications, as a basic risk factor for the development of cardiovascular diseases and acute incidents, is - indirectly - the main cause of death in developed countries. Most of the available scientific studies assessing remodelling of the heart are focused on morphological changes within the left ventricle, which is directly affected by the pathological triggers of this remodelling, e.g. hypertension or cardiomyopathy, which apparently lead to asymmetrical hypertrophy of its walls (interventricular septal wall in comparison to the inferior wall) [19]. Similarly the right ventricle becomes a target of remodelling during pulmonary hypertension of different origins.

Right ventricular hypertrophy due to pulmonary hypertension has got higher attention of the scientists since last few years. However little do we know about already observed, but still sparsely described phenomenon of "cross-remodelling", in this case, within the right ventricle during arterial hypertension [9].

In this study, looking for anatomical exponents of myocardial remodelling within the RV musculature in people with hypertension, we have set ourselves the

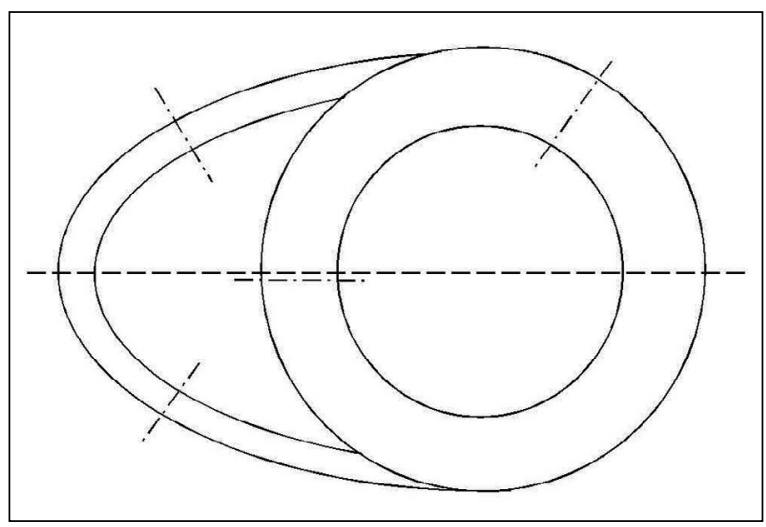

Figure 1. The scheme of heart opening cut (dashed line) and heart walls cuts for measurements (dashed-dot lines).

goal of answering the question whether myocardial remodelling may also involve structures that are not directly affected by the causative agent.

\section{MATERIALS AND METHODS}

The material for this study consisted of 35 human hearts from forensic sections and anatomopathological sections of people who died of non-cardiac causes (e.g. traffic accidents, suicide attempts, strokes, acute infections). All hearts were instantly fixed in a solution of formalin and ethanol. The control group (CG) consisted of 13 hearts ( 6 female [F]/7 male [M]; weight (mean \pm standard deviation [SD]): $317.7 \pm$ $\pm 35.15 \mathrm{~g}$; age (average \pm SD): $50.9 \pm 16.8$ years) from persons in whom no cardiovascular disease has been diagnosed in vivo and no obvious pathological changes were found in post-mortem assessment. The study group (SG) consisted of 22 hearts (10 F/12 M; weight $[$ mean $\pm S D$ ]: $533.2 \pm 68.34 \mathrm{~g}$; age [average \pm SD] : $51.7 \pm 16.8$ years) from people with a history of arterial hypertension and LV hypertrophy found during anatomopathological examination. The protocol was approved by the Independent Bioethics Commission for Research of the Medical University of Gdansk (consent no. NKBBN/165/2018 dated 12 April 2018).

The material was subjected to macro- and microscopic assessment. The right ventricle cavity was opened with a long axis cut along the acute margin from the tricuspid ring to the apex of the right ventricle. Similarly, the left ventricle cavity was opened with a cut along the oblique margin. In addition, the interventricular septum (IVS) was dissected along the long axis, in its frontal plane, in its mid-antero-posterior dimension (Fig. 1). After opening the hearts, the ven- 


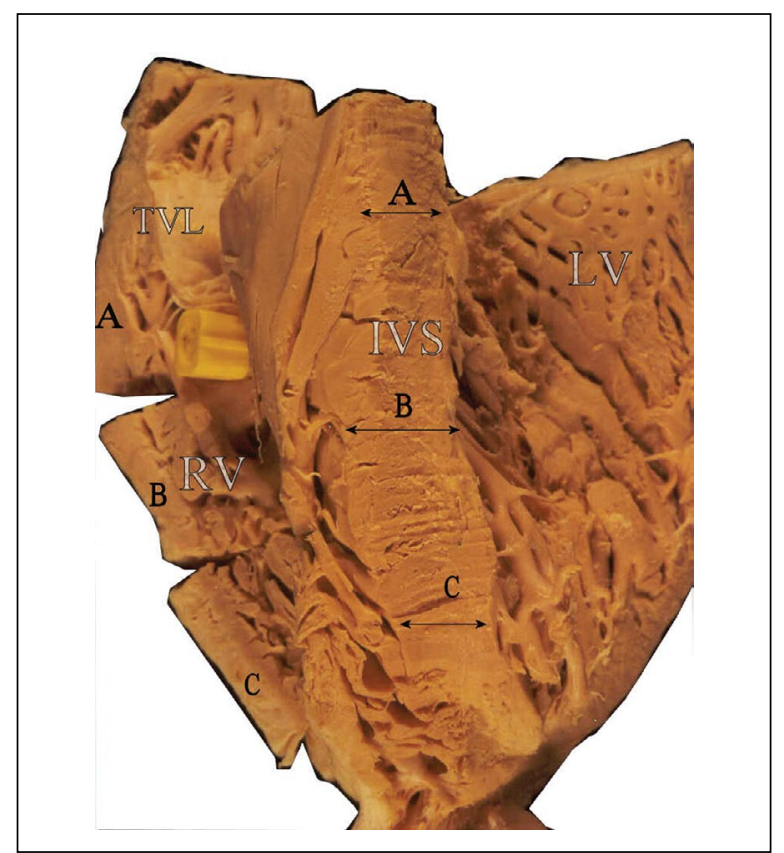

Figure 2. Opened heart and incisions of the walls to take measurements; LV — left ventricle, RV — right ventricle; IVS — interventricular septum; TVL — tricuspid valve leaflet; $A, B, C$ - measurements of heart walls thickness at appropriate levels.

tricular septum was divided into four equal sections, thus determining three levels: A, B and C. At these levels, halfway between the acute margin and the septum (RV) and between the oblique margin and the septum (LV) incisions of the heart walls were made and the thickness of both RV walls, LV inferior wall and IVS were measured. The measurements were designated as dimensions A, B and C, respectively (Fig. 2).

The specimens for microscopic assessment were taken from: RV posterior wall $(1 \times 1 \mathrm{~cm})$, IVS (covering the entire IVS section) and a fragment from the LV inferior wall $(1 \times 1 \mathrm{~cm})$. Seven hearts were randomly selected for this stage of the study from each group (the control group and the study group). In order to reveal various types of tissue within the heart wall, the preparations were subjected to Masson staining with Goldner modification, which allows simultaneous assessment of connective, adipose tissues, and myocardial muscle cells. The preparations were evaluated in the Opta-Tech MN 800 light microscope (Opta-Tech, Poland), with Moticam 2000 2.0M camera (Motic Incorporation Ltd., Hong Kong) and Leica MZ8/MPS60 (Leica Microsystems Ltd., Switzerland) photographic adapter with the use of Motic Images Plus 2.0 software (version 2.02006 Motic China Group Co., Ltd.)

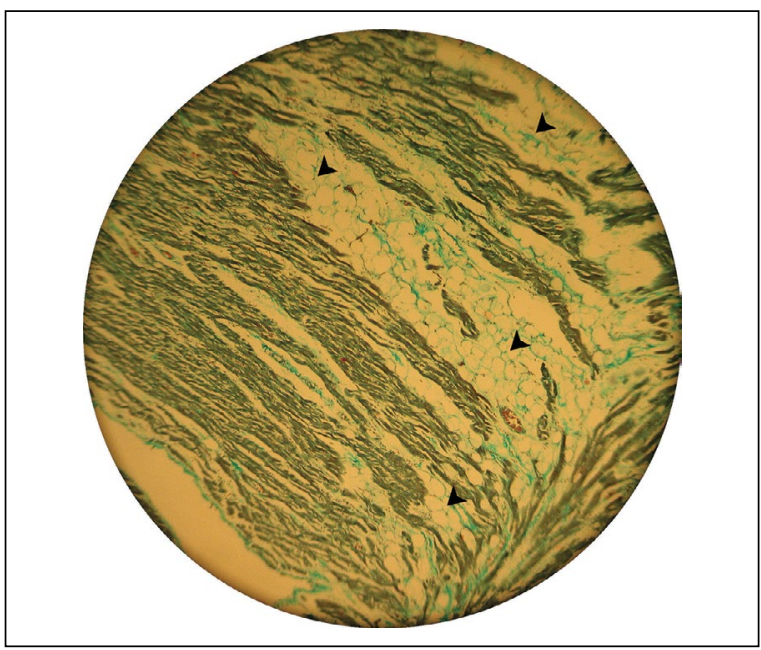

Figure 3. Right ventricle myocardium infiltration by adipose tissue (arrows) (male I.71; Mason-Goldner staining; $62.5 \times$ ).

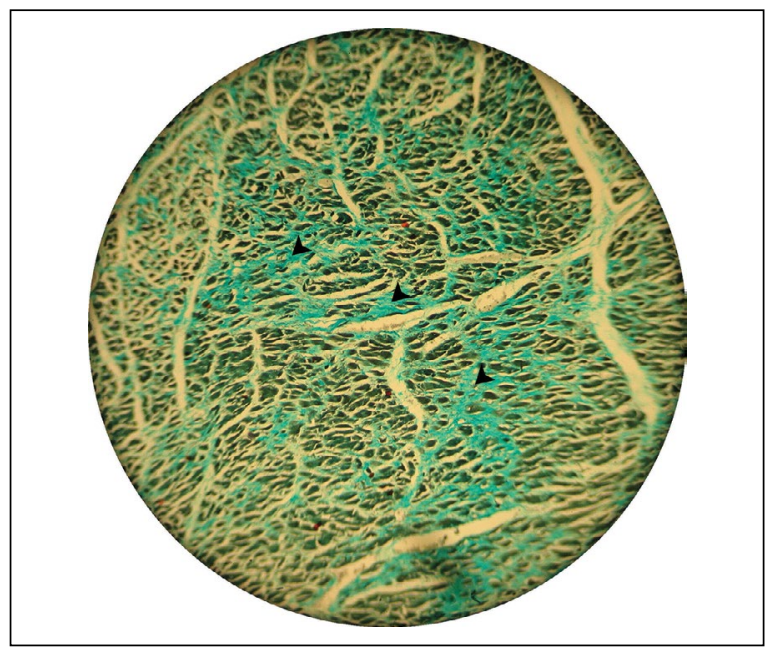

Figure 4. Interstitial fibrosis (arrows) of interventricular septum myocardium (male I.71; Mason-Goldner staining; 62.5×).

During microscopic assessment the following features were evaluated: 1) cardiomyocyte thickness, 2) infiltration of the muscular layer of the heart wall by adipose tissue, which penetrates between the muscle fibre strands, thus separating them from each other (Fig. 3), 3) disturbance of the proportion between the amount of muscle tissue and connective tissue, occurring in the form of clusters; its excessive growth is referred to as interstitial myocardial fibrosis (Fig. 4).

\section{Statistical analysis}

Statistical analysis was performed using Fisher's test and Student's t-test for mean of small samples. 
The significance of differences between the results obtained from the qualitative observations of microscopic changes was confirmed using the non-parametric Mann-Whitney $U$ test for two independent groups. $P$ values $<0.05$ were considered statistically significant. The software used to perform analyses was Statistica 9.0 package from StatSoft.

\section{RESULTS}

\section{Macroscopic evaluation}

\section{Inferior wall of the left ventricle}

Individual dimensions of the inferior wall of the left ventricle, which is the target of systemic hypertension, in SG hearts were significantly larger than those of CG. It is noteworthy that these differences were larger related to dimensions $A$ and $B$, and slightly smaller (but still statistically significant) for dimension C. At level A, the thickness of the LV inferior wall in CG hearts was on average $10.28 \pm 1.24 \mathrm{~mm}$, while in hearts with hypertrophy this average was $14.26 \pm 2.35 \mathrm{~mm}$ $(p<0.000003)$. Dimension B averaged $10.01 \pm$ $\pm 0.95 \mathrm{~mm}$ in CG and $13.27 \pm 2.06 \mathrm{~mm}$ in SG $(p<0.00001)$. Dimension C was $8.71 \pm 1.20 \mathrm{~mm}$ in CG and $10.34 \pm 2.40 \mathrm{~mm}$ in SG ( $p<0.05)$, respectively.

As the effect of the above changes, also the difference in proportions between individual dimensions of the LV inferior wall was noticeable. While there were no differences between the two groups in the $A / B$ and $B / C$ ratios, in hearts with hypertrophy the $A / C$ ratio was significantly higher $(p<0.05)$ than in $C G$. This implies a greater disproportion between dimensions $A$ and $C$ in SG (Table 1).

Assessing the shape of the cross-sectional plane along the long axis of the LV inferior wall, some differences in the geometry of this cross-section were observed. Namely, in CG dimensions A and B were similar and dominated over dimension $C$, which meant that in this group of hearts the cross-section of the LV inferior wall between the levels of the valve attachment $(A)$ and the base of the papillary muscle (B) had a rectangular shape, and below it, towards the apex of left ventricle, it gradually decreased, giving the last segment a trapezoidal shape. In SG, on the other hand, the highest thickness occurred at level A, and then downwards gradually decreased. Therefore, the whole cross-section of the inferior wall assumed the shape of an inverted trapezoid (Table 2).

\section{Interventricular septum}

Same as the LV inferior wall, in terms of individual dimensions $A, B$ and $C$, the thickness of IVS of
Table 1. Comparison of the ratios of the individual dimensions of the inferior wall of the left ventricle in the hearts of control group (CG) and study group (SG)

\begin{tabular}{lccc}
\hline & A/B & B/C & A/C \\
\hline CG & 1.031 & 1.162 & 1.199 \\
SG & 1.044 & 1.467 & 1.521 \\
$\mathrm{p}$ & 0.28 & 0.11 & $\mathbf{0 . 0 4 7}$ \\
\hline
\end{tabular}

$A / B, B / C, A / C$ - relations of the individual dimensions, $p$ - level of statistical significance

Table 2. Schematic representation of the shape of longitudinal section of each of the walls of the heart in control group (CG) and study group (SG)

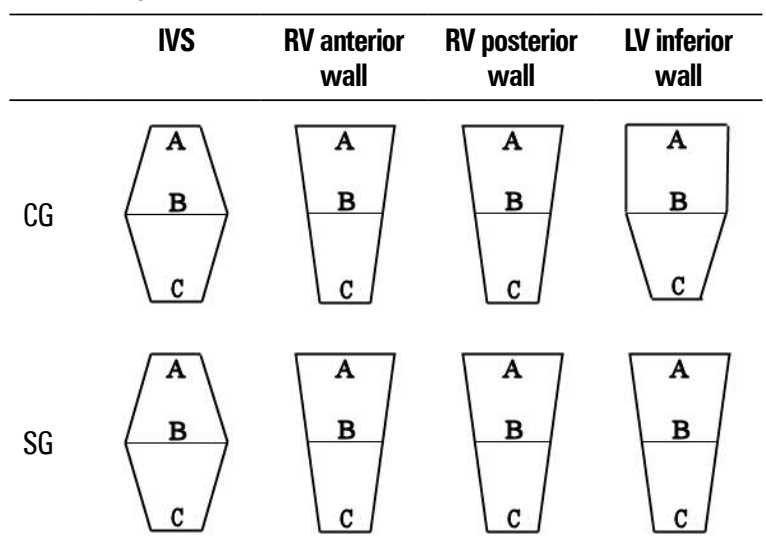

IVS — interventricular septum, RV — right ventricle, LV — left ventricle, CG - control group, SG - study group, A, B, C - levels at which the thickness of the myocardium has been measured

hearts from the SG was significantly greater than that of CG hearts. The average dimension A in CG was $10.6 \pm 1.20 \mathrm{~mm}$, while in SG it was $13.4 \pm 2.15 \mathrm{~mm}$ $(p=0.0001)$. Dimension B was $11.6 \pm 1.18 \mathrm{~mm}$ and $15.93 \pm 2.52 \mathrm{~mm}$ in groups CG and SG, respectively $(p<0.0001)$. Dimension C averaged $9.91 \pm 1.52 \mathrm{~mm}$ in $C G$, and $12.25 \pm 2.67 \mathrm{~mm}$ in SG ( $p=0.006)$.

However, despite such a significant differentiation between individual dimensions in both groups, the comparison of ratios of these dimensions $(A / B, B / C$, $A / C)$ did not show statistically significant deviations. It seems that hypertrophy of the heart muscle within the septum occurs evenly at all its levels, leading to a proportional increase in all measured IVS dimensions (Table 3).

\section{Anterior wall of the right ventricle}

As in the case of the in LV inferior wall, significant differences in the thickness of the muscle between both groups were also observed within the anterior wall of the right ventricle. The RV anterior wall 
Table 3. Comparison of the ratios of the individual dimensions of the interventricular septum in the hearts of control group (CG) and study group (SG)

\begin{tabular}{lccc}
\hline & $\mathbf{A} / \mathbf{B}$ & $\mathbf{B} / \mathbf{C}$ & $\mathbf{A} / \mathbf{C}$ \\
\hline $\mathrm{CG}$ & 0.917 & 1.192 & 1.093 \\
$\mathrm{SG}$ & 0.843 & 1.260 & 1.066 \\
$\mathrm{p}$ & 0.09 & 0.07 & 0.68 \\
\hline
\end{tabular}

$A / B, B / C, A / C$ - relations of the individual dimensions; $p$ - level of statistical significance

Table 4. Comparison of the ratios of the individual dimensions of the anterior wall of the right ventricle in the hearts of control group (CG) and study group (SG)

\begin{tabular}{lccc}
\hline & A/B & B/C & A/C \\
\hline CG & 1.406 & 1.360 & 1.866 \\
SG & 1.274 & 1.351 & 1.761 \\
$\mathrm{p}$ & 0.62 & 0.97 & 0.97 \\
\hline
\end{tabular}

$A / B, B / C, A / C$ - relations of the individual dimensions; $p$ - level of statistical significance

Table 5. Comparison of the ratios of the individual dimensions of the posterior wall of the right ventricle in the hearts of control group (CG) and study group (SG)

\begin{tabular}{lccc}
\hline & A/B & B/C & A/C \\
\hline CG & 1.097 & 1.514 & 1.676 \\
SG & 1.142 & 1.384 & 1.462 \\
$\mathrm{p}$ & 0.76 & 0.94 & 0.72 \\
\hline
\end{tabular}

$A / B, B / C, A / C$ - relations of the individual dimensions; $p$ - level of statistical significance

average thickness at level $A$ was $4.35 \pm 0.63 \mathrm{~mm}$ in CG, while in SG $5.35 \pm 1.00 \mathrm{~mm}(p<0.001)$. The average dimension $B$ was $3.18 \pm 0.72 \mathrm{~mm}$ in $\mathrm{CG}$ and $4.19 \pm 1.16 \mathrm{~mm}$ in SG $(p<0.005)$. Dimension $C$, measured near the apex of the right ventricle, was on average $2.37 \pm 0.40 \mathrm{~mm}$ and $3.20 \pm 1.20 \mathrm{~mm}$ in CG and SG, respectively ( $p<0.05)$.

No statistically significant differences were noted between the two groups assessing the proportions between individual dimensions (Table 4).

The thickness of the anterior wall at individual levels was typical in all hearts with the largest dimension A, intermediate B and the smallest dimension C. Therefore, the longitudinal section through this wall gives the shape of a triangle, with the base pointing towards the base of the heart, and the top pointing towards the apex of the right ventricle. This image was the same for both groups of hearts (Table 2).

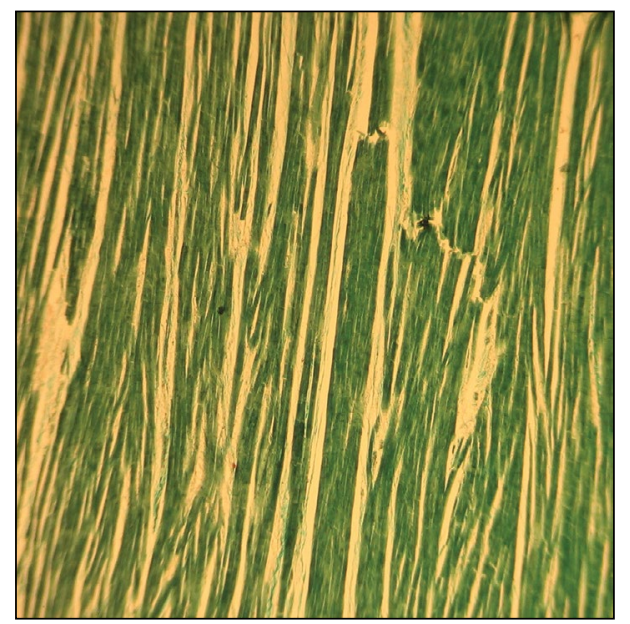

Figure 5. Microscopic view of a healthy myocardium of interventricular septum (male I.24; Mason-Goldner staining; $62.5 \times$ ).

\section{Posterior wall of the right ventricle}

Within the RV posterior wall, statistically significant differences were also noted for individual dimensions $A, B$ and $C$. The average dimension $A$ in CG was $4.27 \pm 0.89 \mathrm{~mm}$, while in SG it was $5.11 \pm$ $\pm 1.28 \mathrm{~mm}(p<0.05)$. Dimension B was $3.93 \pm 0.69$ $\mathrm{mm}$ and $4.73 \pm 1.17 \mathrm{~mm}$, in CG and SG, respectively $(p<0.05)$. In CG the dimension $C$ averaged $2.68 \pm$ $\pm 0.81 \mathrm{~mm}$, and in SG $3.38 \pm 1.18 \mathrm{~mm}(p<0.05)$.

Again, despite the differences observed, comparing the ratios of these dimensions $(A / B, B / C, A / C)$ in both groups did not reveal any statistically significant deviations (Table 5).

The posterior wall of the right ventricle in its longitudinal section had, like the RV anterior wall, the shape of an inverted triangle, resulting directly from its dimensions and their proportions (Table 2).

\section{Microscopic evaluation}

In the CG, the average thickness of cardiomyocytes within the RV and LV free walls as well as IVS was $10 \mu \mathrm{m}$. In 1 (14.3\%) case, fat infiltration into the muscular layer of the RV wall was observed. This phenomenon did not occur in the remaining examined walls of this heart, nor in any of the walls of other hearts in this group (Fig. 5). Also in 1 (14.3\%) case (same heart), interstitial fibrosis was noted, which was present in all its walls (RV, LV and IVS). Other hearts in this group did not show this pathology. In the vast majority of cases, the muscle fibre arrangement was regular. Only in 3 hearts ( 2 hearts - RV wall, 1 heart 
Table 6. Table of microscopic observations - frequency [\%] of morphological features of remodelling of the heart muscle in control group and study group

\begin{tabular}{|c|c|c|c|c|c|c|c|c|c|}
\hline \multirow[t]{2}{*}{ Group } & \multicolumn{3}{|c|}{ Adipose tissue infiltrating myocardium [\%] } & \multicolumn{3}{|c|}{ Interstitial fibrosis [\%] } & \multicolumn{3}{|c|}{ Average myocytes thickness [ $\mu \mathrm{m}]$} \\
\hline & RV & IVS & LV & RV & IVS & LV & RV & IVS & $\mathbf{L V}$ \\
\hline CG & 14.3 & 0 & 0 & 14.3 & 14.3 & 14.3 & 10 & 10 & 10 \\
\hline SG & 14.3 & 0 & 0 & 14.3 & 0 & 14.3 & 10 & 13.5 & 14 \\
\hline
\end{tabular}

CG — control group, no hypertrophy; SG — study group, with myocardial hypertrophy; RV — posterior wall of the right ventricle; IVS — interventricular septum; LV — inferior wall of the left ventricle

- IVS; $28.6 \%$ and $14.3 \%$, respectively), incidents of area-limited disturbance of the cardiomyocytes co-axial arrangement could be observed.

In the SG with macroscopic hypertrophy of the heart muscle, apart from the assessment of cardiomyocyte thickness, the results of microscopic observations did not differ much from those obtained in the assessment of CG. Within free walls of the RV, in all cases, the average diameter was $10 \mu \mathrm{m}$. Within the remaining cardiac walls (IVS and LV), cardiomyocytes most often reached a diameter ranging 12-15 $\mu \mathrm{m}$. In one of the examined hearts in SG, the presence of adipose tissue was observed only in the RV musculature (14.3\%). The other walls of this heart, as well as other walls of remaining hearts, did not show the presence of this feature. Interstitial fibrosis was observed in the same heart, also within RV (14.3\%), and in the second heart, only in the muscle of the left ventricle (14.3\%). The other walls in these 2 cases, as well as other hearts, also did not show the presence of this feature. Despite the myocardial hypertrophy found at the stage of macroscopic examination, microscopic examination did not show any disturbance of the spatial arrangement of muscle fibres in any of these hearts (Table 6).

\section{DISCUSSION}

Remodelling of the heart muscle is a phenomenon quite well known and described. The natural course and final effect of this remodelling at organ, tissue and cellular levels may look different depending on the initiating factor. There are many reports focusing on a single heart chamber myocardial remodelling, e.g.: LV muscle under the influence of ischaemic disease and myocardial infarction [8, 21], valvular defects of the left arterial and atrioventricular outlet [15], cardiomyopathy [13], arterial hypertension [18]; RV musculature in chronic pulmonary hypertension of various aetiologies [11] or chronic obstructive pulmonary disease [2].

However, only recent years have resulted in reports of researchers who drew attention to the fact that in the heart, which is obviously one inseparable whole, there is a possibility of adverse phenomena within the myocardial cavity seemingly unrelated to a given aggravating factor.

Research conducted by Tadic et al. [23-25] has shown a clear correlation between the occurrence of arterial hypertension and remodelling of the RV muscle, which leads to impairment of its function. The above observations were also reflected in the work conducted by Cuspidi et al. [5, 6] and other researchers $[3,26]$.

Interestingly, changes indicating this remodelling were observed not only in the group of patients with arterial hypertension, but also at earlier stages of the disease [17], and even in the group of patients with arterial blood pressure classified as "normal high" [16, 22].

The above studies were based on in vivo echocardiographic assessment of right ventricle morphology and function. Our study seems to partially confirm these observations on the sectional material.

It may be argued that statistically significant changes in the thickness of the free wall of the right heart ventricle do not go hand in hand with evident cardiomyocyte hypertrophy. However, if we take into account the fact that although the thickness of the RV free walls in SG compared to CG was significantly greater, it still remained within the accepted echocardiographic norms (up to $5 \mathrm{~mm}$ ), exceeding them only at level $A$.

It can be suspected that in SG the phenomenon occurring in the RV musculature is a result of regulatory and/or compensatory processes occurring in the myocardium as a whole and may be equivalent to physiological hypertrophy observed within the LV muscle in conditions of its increased physiological load (e.g. practicing sports, pregnancy). This thesis is supported by the fact that microscopic examination revealed no evidence of permanent, irreversible remodelling of the RV muscle.

As the authors of this study we are aware of its undoubted limitation of the small size of the groups, 
which limits both the possibility of formulating fully convincing conclusions and the effectiveness of statistical methods. Unfortunately, the selection of material originating from people burdened only with hypertension (in order to eliminate other known factors that can lead to muscle remodelling of any of the walls of the heart) and who died of non-cardiovascular causes is an extremely problematic task. Difficulties associated with obtaining this type of selected material is extremely difficult and time-consuming and affected its limited number.

\section{CONCLUSIONS}

Although in vivo echocardiographic tests as well as our macroscopic evaluation of post-mortem material suggest such suspicions, based on the results of the study (primarily the microscopic observations), it was not possible to answer the question of whether the $\mathrm{RV}$ muscle remodelling is evident during hypertension. Instead of a simple, unambiguous answer, the question arises: why and how the RV systolic and diastolic function is disturbed, sometimes at a very early stage of the development of arterial hypertensive disease. Confirmation of this requires further research, possibly reaching deeper into the ultrastructure of the cardiomyocyte.

\section{Conflict of interest: None declared}

\section{REFERENCES}

1. Anrep GV, Häusler $H$. The coronary circulation: I. The effect of changes of the blood-pressure and of the output of the heart. J Physiol. 1928; 65(4): 357-373, doi: 10.1113/jphysiol.1928. sp002481, indexed in Pubmed: 16993957.

2. Biernacki W, Flenley DC, Muir AL, et al. Pulmonary hypertension and right ventricular function in patients with COPD. Chest. 1988; 94(6): 1169-1175, doi: 10.1378/chest.94.6.1169, indexed in Pubmed: 3191757.

3. Cicala S, Galderisi M, Caso P, et al. Right ventricular diastolic dysfunction in arterial systemic hypertension: analysis by pulsed tissue Doppler. Eur J Echocardiogr. 2002; 3(2): 135-142, doi: 10.1053/ euje.2001.0124, indexed in Pubmed: 12114098.

4. Cohn JN. Structural basis for heart failure. Ventricular remodeling and its pharmacological inhibition. Circulation. 1995; 91(10): 2504-2507, doi: 10.1161/01.cir.91.10.2504, indexed in Pubmed: 7743609.

5. Cuspidi C, Sampieri L, Angioni L, et al. Right ventricular wall thickness and function in hypertensive patients with and without left ventricular hypertrophy: echo-Doppler study. J Hypertens Suppl. 1989; 7(6): S108-S109, doi: 10.1097/00004872-19890007600050, indexed in Pubmed: 2534401.

6. Cuspidi C, Valerio C, Sala C, et al. Prevalence and correlates of left atrial enlargement in essential hypertension: role of ventricular geometry and the metabolic syndrome: the Evaluation of Target Organ Damage in $\mathrm{Hy}-$ pertension study. J Hypertens. 2005; 23(4): 875-882, doi: 10.1097/01. hjh.0000163158.14493.23, indexed in Pubmed: 15775794.

7. D'Andrea A, Caso P, Scarafile R, et al. Biventricular myocardial adaptation to different training protocols in competitive master athletes. Int J Cardiol. 2007; 115(3): 342-349, doi: 10.1016/j. ijcard.2006.03.041, indexed in Pubmed: 16959340.

8. Dong L, Mintz GS, Witzenbichler B, et al. Comparison of plaque characteristics in narrowings with ST-elevation myocardial infarction (STEMI), non-STEMI/unstable angina pectoris and stable coronary artery disease (from the ADAPT-DES IVUS Substudy). Am J Cardiol. 2015; 115(7): 860-866, doi: 10.1016/j.amjcard.2015.01.008, indexed in Pubmed: 25661569.
9. Franco V. Right ventricular remodeling in pulmonary hypertension. Heart Fail Clin. 2012; 8(3): 403-412, doi: 10.1016/j. hfc.2012.04.005, indexed in Pubmed: 22748902.

10. Grajek S. Patofizjologia przebudowy serca. In: Szyszka A (ed.). Przebudowa serca. ViaMedica, Gdańsk 2002: 1-30.

11. Harrison A, Hatton N, Ryan JJ. The right ventricle under pressure: evaluating the adaptive and maladaptive changes in the right ventricle in pulmonary arterial hypertension using echocardiography (2013 Grover Conference series). Pulm Circ. 2015; 5(1): 29-47, doi: 10.1086/679699, indexed in Pubmed: 25992269.

12. Kamiński L, Płońska E, Szyszka A, et al. [Echocardiographic examination of cardiac structure and function in male athletes of static and dynamic disciplines]. Pol Merkur Lek. 2006; 20(117): 274-278, indexed in Pubmed: 16780253.

13. Latus H, Gummel K, Klingel K, et al. Focal myocardial fibrosis assessed by late gadolinium enhancement cardiovascular magnetic resonance in children and adolescents with dilated cardiomyopathy. J Cardiovasc Magn Reson. 2015; 17: 34, doi: 10.1186/ s12968-015-0142-0, indexed in Pubmed: 25976093.

14. Limbourg $P$, Wende $W$, Henrich $H$, et al. [Frequency potentiation and Frank-Starling mechanism in canine ventricle under sinus node rhythm and ventricular pacing]. Pflugers Arch. 1971; 322(3): 250-263, doi: 10.1007/BF00602073, indexed in Pubmed: 5099687.

15. Park JiY, Ryu SK, Choi JW, et al. Association of inflammation, myocardial fibrosis and cardiac remodelling in patients with mild aortic stenosis as assessed by biomarkers and echocardiography. Clin Exp Pharmacol Physiol. 2014; 41(3): 185-191, doi: 10.1111/14401681.12206, indexed in Pubmed: 24471798.

16. Pedrinelli R, Canale ML, Giannini C, et al. Abnormal right ventricular mechanics in early systemic hypertension: a two-dimensional strain imaging study. Eur J Echocardiogr. 2010; 11(9): 738-742, doi: 10.1093/ejechocard/jeq059, indexed in Pubmed: 20472915.

17. Pedrinelli $R$, Canale ML, Giannini $C$, et al. Right ventricular dysfunction in early systemic hypertension: a tissue Doppler imaging study in patients with high-normal and mildly increased arterial blood pressure. J Hypertens. 2010; 28(3): 615-621, doi: 10.1097/ hjh.0b013e328334f181, indexed in Pubmed: 20191674.

18. Puchades R, Ruiz-Nodar JM, Blanco F, et al. An analysis of cardiac remodeling in the elderly population. EPICARDIAN study. Rev Esp Cardiol. 2010; 63(8): 989-991, doi: 10.1016/s18855857(10)70193-6, indexed in Pubmed: 20738944.

19. Rodrigues JCL, Amadu AM, Dastidar AG, et al. Prevalence and predictors of asymmetric hypertensive heart disease: insights from cardiac and aortic function with cardiovascular magnetic resonance. Eur Heart J Cardiovasc Imaging. 2016; 17(12): 1405-1413. doi: 10.1093/ehjci/jev329, indexed in Pubmed: 26705488.

20. Schannwell CM, Zimmermann T, Schneppenheim M, et al. Left ventricular hypertrophy and diastolic dysfunction in healthy pregnant women. Cardiology. 2002; 97(2): 73-78, doi: 10.1159/000057675, indexed in Pubmed: 11978952.

21. Schuleri $\mathrm{KH}$, Centola $\mathrm{M}$, Evers $\mathrm{KS}$, et al. Cardiovascular magnetic resonance characterization of peri-infarct zone remodeling following myocardial infarction. J Cardiovasc Magn Reson. 2012; 14: 24, doi: 10.1186/1532-429X-14-24, indexed in Pubmed: 22510220.

22. Tadic M, Cuspidi C, Pencic B, et al. High-normal blood pressure impacts the right heart mechanics: a three-dimensional echocardiography and two-dimensional speckle tracking imaging study. Blood Press Monit. 2014; 19(3): 145-152, doi: 10.1097/ MBP.0000000000000043, indexed in Pubmed: 24695214.

23. Tadic M, Cuspidi C, Pencic B, et al. Relationship between right ventricular remodeling and heart rate variability in arterial hypertension. J Hypertens. 2015; 33(5): 1090-1097, doi: 10.1097/ HJH.0000000000000511, indexed in Pubmed: 25668353.

24. Tadic M, Ivanovic B, Celic V, et al. Are the metabolic syndrome, blood pressure pattern, and their interaction responsible for the right ventricular remodeling? Blood Press Monit. 2013; 18(4): 195-202, doi: 10.1097/MBP.0b013e3283631af4, indexed in Pubmed: 23777905.

25. Tadic M, Ivanovic B, Celic $V$, et al. The impact of metabolic syndrome, recently diagnosed diabetes and hypertension on right ventricular remodeling. Is there difference between risk factors? Clin Exp Hypertens. 2014; 36(5): 295-301, doi: 10.3109/10641963.2013.810235, indexed in Pubmed: 23865506.

26. Tumuklu MM, Erkorkmaz U, Ocal A. The impact of hypertension and hypertension-related left ventricle hypertrophy on right ventricle function. Echocardiography. 2007; 24(4): 374-384, doi: 10.1111/j.1540-8175.2007.00419.x, indexed in Pubmed: 17381646.

27. Weber KT, Sun Y, Guarda E. Structural remodeling in hypertensive heart disease and the role of hormones. Hypertension. 1994; 23(6 Pt 2): 869-877, doi: 10.1161/01.hyp.23.6.869, indexed in Pubmed: 8206620. 